PAPER

\title{
On the overlap between apathy and depression in dementia
}

\author{
S E Starkstein, L Ingram, M L Garau, R Mizrahi
}

J Neurol Neurosurg Psychiatry 2005;76:1070-1074. doi: 10.1136/jnnp.2004.052795

See end of article for authors' affiliations

....................

Correspondence to: Dr Sergio E Starkstein, Fremantle Hospital, Education Building T-7, Fremantle WA 6959, Australia; ses@cyllene. uwa.edu.au

Received 26 August 2004 Revised version received 17 October 2004 Accepted

17 November 2004

\begin{abstract}
Background: Whereas apathy is increasingly recognised as a frequent abnormal behaviour in dementia, its overlap with depression remains poorly understood.

Aims: To assess the psychometric characteristics of a structured interview for apathy, and to examine the overlap between apathy and depression in dementia.

Methods: A total of 150 patients with Alzheimer's disease (AD) underwent a comprehensive psychiatric and cognitive assessment.

Results: Twelve per cent of the sample met criteria for both apathy and depression, $7 \%$ met criteria for apathy only, and $31 \%$ met criteria for depression only. Apathy (but not depression) was significantly associated with more severe cognitive deficits. Apathy and anxiety scores accounted for $65 \%$ of the variance of depression scores in dementia, and the diagnosis of apathy had a minor impact on the rating of severity of depression.

Conclusions: The Structured Interview for Apathy demonstrated adequate psychometric characteristics. Using a novel structured interview for apathy in $A D$ we demonstrated that whereas the construct of depression primarily consists of symptom clusters of apathy and anxiety, apathy is a behavioural dimension independent of depression.
\end{abstract}

W hereas apathy is increasingly recognised as a frequent behavioural problem in dementia, its nosological position remains unclear. The philosophical meaning of apathy is "lack of passion", the latter being an extreme form of emotion. ${ }^{1}$ The Stoic philosophers aimed at being free of passions, given that in their conception only a state of true apathy would release the human mind from emotional constraints. The contemporary psychiatric meaning of apathy is obviously different from the Stoic and more akin to the concept of "abulia", which is defined as "loss, lack or impairment of the power to will or execute what is in mind".

Kraepelin defined manic depressive insanity as a state of abnormal volition, emotion, and cognition, and this conception is included in contemporary psychiatric nomenclatures. ${ }^{3}$ The modern concepts of apathy and depression both share the predicate of "reduced volition" (in the etymological sense of "acting an intention"), which automatically implies a phenomenological overlap. Depression could still occur in the absence of apathy, provided depressed individuals show mood-congruent emotional changes, whereas apathy could occur in the absence of depression provided the changes in volition do not co-occur with the emotional changes of depression.

The frequencies of apathy and depression in dementia were reported to vary widely, and different phenomenological conceptions of these constructs may explain discrepant findings. ${ }^{45}$ Another confounding factor is that, to our knowledge, structured instruments to diagnose apathy have not been developed, and apathy has been mostly diagnosed using arbitrary cut off scores on ad hoc severity rating scales. Main aims for the present study were to assess the psychometric characteristics of the newly designed Structured Interview for Apathy, to diagnose apathy in dementia based on standardised criteria, and to examine the phenomenological overlap between apathy and depression.

\section{METHOD}

\section{Patients}

Alzheimer's disease group

The Alzheimer's disease (AD) group consisted of 154 consecutive patients with progressive cognitive decline who visited the dementia clinic at a large tertiary clinic centre in
Buenos Aires, Argentina. The patients met the National Institute of Neurological and Communicative Disorders and Stroke and the Alzheimer's Disease and Related Disorders Association criteria for probable AD. ${ }^{6}$ None of the patients had a history of stroke, evidence of focal lesions on a magnetic resonance imaging brain scan ( $\mathrm{Tl}$ weighted), or a Hachinski ischemic score $>4$. $^{6}$

\section{Psychiatric examination}

After the methodology of the study had been fully explained, written informed consent was obtained from the patients and their respective caregivers. Four patients declined participation. The evaluation included administration of the Structured Clinical Interview for DSM-IV (SCID), ${ }^{7}$ a semi-structured diagnostic interview for assessing signs and symptoms necessary for the major axis I DSM-IV diagnoses; the Mini-Mental State Exam, ${ }^{8}$ a global measure of cognitive deficits; the Hamilton Depression Rating Scale, ${ }^{9}$ a 17 item interviewer rated scale for rating the severity of symptoms of depression; the Hamilton Anxiety Rating Scale, ${ }^{10}$ a 14 item interviewer rated scale for rating the severity of symptoms of anxiety; the Clinical Dementia Rating, ${ }^{11}$ a global rating device for dementia stages; and the Irritability and Apathy Scales, 14 item scales for rating the severity of symptoms of irritability and apathy, respectively. ${ }^{12}$ Patients with $\mathrm{AD}$ were interviewed first. Simultaneously, caregivers, who were blind to the results of these interviews, rated the patients' behaviours with the corresponding instruments. Finally, the psychiatrist administered the SCID and the Structured Interview for Apathy (described below) to each patient, with both the patient and the caregiver present. We previously demonstrated the reliability and validity of these instruments in dementia. ${ }^{12}{ }^{13}$

\section{Diagnosis of apathy}

We designed the Structured Interview for Apathy to screen for symptoms of apathy as operationalised by Marin ${ }^{14}$ and Starkstein et $a l^{13}$ into clinical diagnostic criteria. The

Abbreviations: $A D$, Alzheimer's disease; CAMDEX, Cambridge Examination for Mental Disorders of the Elderly; MMSE, Mini-Mental State Examination; SCID, Structured Clinical Interview for DSM-IV 
Structured Interview for Apathy includes questions assessing the domains of lack of motivation relative to the individual's previous level of functioning, lack of effort to perform every day activities, dependency on others to structure activity, lack of interest in learning new things or in new experiences, lack of concern about one's personal problems, unchanging or flat affect, and lack of emotional response to positive or negative personal events. All questions were structured using the SCID format. Each criterion is assessed with two key questions, and additional follow up questions are used to rate the severity of symptoms. Criterion A (lack of motivation relative to the patient's previous level of functioning) is assessed with the following questions: "Did you notice a lack of or diminished motivation to perform the activities of daily living? Does it happen most of the day, almost every day?". These questions are followed by specific questions to rate the frequency of the symptom, the approximate date of onset, the pattern of progression, and discrepancies in the information provided by patient and caregiver. All these questions are assessed for all the subsequent positive responses for each of the following items: criterion Bl (lack of effort or energy to perform everyday activities (for example, grooming, work, social life): "Did you notice lack of or putting less effort into your everyday activities? If an extra effort is needed do you refrain from doing things? Are you less helpful in household chores?"; criterion B2 (dependency on prompts from others to carry out everyday activities): "Do you feel that in order to carry out your daily activities you need that someone indicates or organises your activities? Do you need a "push" to start doing your daily activities?"; criterion B3 (lack of interest in learning new things, or in new experiences): "Do you feel that you have less interest to learn new things? (for example, reading newspapers, books, watching TV)? Do you note less interest to learn about events in your environment, such as family meetings, neighbourhood news?"; criterion B4 (lack of concern about one's personal problems): "Are you indifferent or less interested in your personal situation? Are you indifferent to your health condition, working situation, hobbies, or personal care?"; and criterion B5 (unchanging or flat affect, lack of emotional response to positive or negative events): "Do you have a flat mood, with little or no fluctuations? Do you feel indifferent in your emotions, neither happy nor sad about positive or negative events in your environment?". Based on answers to these questions, symptoms are scored as either absent, subclinical, or definitely present. Criterion $\mathrm{C}$ assesses the extent of social and occupational dysfunction caused by the symptoms of apathy, and criterion D excludes organic causes of behavioural changes other than dementia. Apathy is diagnosed whenever patients had a score of 3 (that is, "definitely

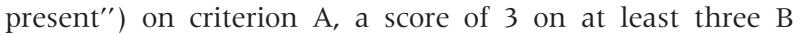
criteria, and a score of 1 (that is, absent) on criteria C and D.

\section{Statistical analysis}

Statistical analysis was carried out with the use of means and standard deviations, two way analysis of variance (ANOVA), and Tukey's post hoc tests. A principal components factor analysis for the Hamilton Depression Rating Scale was carried out using varimax rotation. Frequency distributions were calculated with $\chi^{2}$ and Fisher's exact tests. All $p$ values are two tailed.

\section{RESULTS}

\section{Psychometric characteristics of the structured interview for apathy}

Test-retest reliability was calculated by a single examiner, who assessed 10 patients (four with and six without an initial diagnosis of apathy), 2-4 weeks apart. Diagnostic agreement between the initial and follow up evaluations was perfect
(100\%). Inter-rater reliability was assessed in an additional series of 10 patients (three with and seven without apathy), in separate interviews (2-4 weeks apart) carried out by two different examiners blind to each other's diagnosis. There was perfect $(100 \%)$ diagnostic agreement between examiners. The Structured Interview for Apathy had high internal consistency (Cronbach's $\alpha=0.91$ ), indicating substantial homogeneity among the items. Based on information obtained with the Structured Interview for Apathy, patients meeting diagnostic criteria for apathy $(n=29)$ had a significantly higher mean (SD) score on the caregiver's rated Apathy Scale than patients without apathy $(\mathrm{n}=121): 24.1$ (9.1) v 15.5 (8.1), respectively; $\mathrm{t}=4.74, \mathrm{df}=148, \mathrm{p}<0.0001$.

\section{Demographic and clinical findings}

Twenty nine (19\%) of the 150 patients met Marin's diagnostic criteria for apathy. Thirteen (45\%) of the 29 patients with apathy also had major depression, five patients ( $17 \%$ ) had minor depression, and 11 patients (38\%) were not depressed. Among patients without a clinical diagnosis of apathy, 17 (14\%) had major depression, 30 (25\%) had minor depression, and $74(61 \%)$ had no depression $\left(\chi^{2}=13.8\right.$, $\mathrm{df}=2, \mathrm{p}<0.001)$. Patients with apathy had similar age, years of education, and duration of illness to patients without apathy (table 1).

To examine different clinical correlates of depression and apathy, we calculated two way ANOVAs using a $2 \times 2$ factorial design, which included patients with apathy and major depression $(n=13)$, patients with apathy but no depression $(\mathrm{n}=11)$, patients with major depression but no apathy $(\mathrm{n}=17)$, and patients with neither major depression nor apathy $(\mathrm{n}=74)$ (due to the small sample, patients with minor depression (with or without apathy) were excluded from this comparison). A two way ANOVA (depression by apathy) for Mini-Mental State Examination (MMSE) scores (three patients had missing scores) showed a significant effect for apathy $(\mathrm{F}=9.36, \mathrm{df}=1,108, \mathrm{p}<0.01)$ : patients with apathy (with or without depression) had significantly more severe cognitive deficits than patients without apathy. There was neither a significant effect for depression nor a significant depression by apathy interaction.

\section{Phenomenology of apathy and depression in dementia}

We next examined whether symptoms of apathy may be construed as a specific sub-syndrome within the spectrum of depression in dementia, and to this end we calculated a factor analysis on the 17 item Hamilton Depression Rating Scale. To reduce the number of variables, the items of loss of appetite and weight, and the items of early, middle, and late insomnia were collapsed into single variables of appetite and sleep disturbances, respectively. Two factors were obtained. The first factor loaded $(>0.50)$ on the items of loss of interest, psychomotor retardation, agitation, loss of energy, and loss of appetite/weight (eigenvalue: 4.70, variance: 33\%) and was construed as an apathy factor. The second factor loaded on the items of depressed mood, feelings of guilt, suicidal ideation, psychic anxiety, and insomnia (eigenvalue: 1.23, variance: $9 \%$ ) and was construed as a sadness/anxiety factor.

To examine the clinical correlates of each factor, we calculated a stepwise regression analysis with each factor as the dependent variable, and age, years of education, stage of illness, and scores of cognition, anxiety, and apathy as the independent variables (11 patients with missing values had to be excluded from this analysis). Factor 1 (apathy) showed a significant overall correlation $\left(\mathrm{R}^{2}=0.45, \mathrm{df}=3,127\right.$, $\mathrm{p}<0.0001$ ), and the variables that accounted for a significant part of the variance were the apathy scores $\left(R^{2}=0.32\right.$, $\mathrm{p}<0.0001)$, anxiety scores $\left(\mathrm{R}^{2}=0.08, \mathrm{p}<0.0001\right)$ (that is, 
Table 1 Demographic and clinical characteristics of consecutive patients with probable AD who did and did not meet criteria for apathy and depression*

\begin{tabular}{|c|c|c|c|c|}
\hline & $\begin{array}{l}\text { Patients with neither apathy } \\
\text { nor depression }(n=74)\end{array}$ & $\begin{array}{l}\text { Patients with apathy and } \\
\text { without depression }(n=11)\end{array}$ & $\begin{array}{l}\text { Patients with depression and } \\
\text { without apathy }(n=47)\end{array}$ & $\begin{array}{l}\text { Patients with both apathy and } \\
\text { depression }(n=18)\end{array}$ \\
\hline & $n(\%)$ & $\mathrm{n}(\%)$ & $n(\%)$ & $n(\%)$ \\
\hline Female gender & $44(59)$ & $5(45)$ & $33(70)$ & $12(67)$ \\
\hline Major depression† & & & $17(36)$ & $13(72)$ \\
\hline Minor depression & & & $30(64)$ & $5(28)$ \\
\hline Medications received & & & & \\
\hline Neuroleptics & $3(4)$ & $2(18)$ & $6(13)$ & $5(28)$ \\
\hline Antidepressants & $5(7)$ & $2(18)$ & $6(13)$ & $6(33)$ \\
\hline Anxiolytics & $8(11)$ & 1 (9) & $14(30)$ & $9(50)$ \\
\hline & Mean (SD) & Mean (SD) & Mean (SD) & Mean (SD) \\
\hline Age (years) & $69.2(10.2)$ & 71.8 (13.8) & $71.8(10.0)$ & $71.6(8.2)$ \\
\hline Apathy Scale score & & & & \\
\hline Caregiver rating & $12.1(7.2)$ & $20.3(10.5)$ & $22.2(6.2)$ & $30.2(4.2)$ \\
\hline Patient ratings & $5.8(7.0)$ & $17.7(9.6)$ & $16.1(9.4)$ & $27.9(7.4)$ \\
\hline Hamilton Depression Scale & $5.5(4.5)$ & $7.6(3.9)$ & $18.0(9.2)$ & $22.6(8.2)$ \\
\hline Hamilton Anxiety Scale** & $5.7(4.4)$ & $5.7(4.3)$ & $14.0(7.6)$ & $19.2(11.4)$ \\
\hline MMSE score†† & $24.2(5.7)$ & $20.6(5.3)$ & $23.5(4.5)$ & $19.0(2.8)$ \\
\hline $\begin{array}{l}\text { *Apathy was diagnosed using } \\
\text { Structured Clinical Interview } \mathrm{f} \\
\text { Significant difference between } \\
\mathrm{p}<0.0001 ;{ }^{*} \mathrm{~F}=13.2, \mathrm{df}=3 \text {, } \\
\text { MMSE, Mini-Mental State Exc }\end{array}$ & $\begin{array}{l}g \text { the Structured Interview for } \\
\text { or DSM-IV (SCID). } \\
\text { groups: } \dagger \chi^{2}=13.8, d f=2, p \\
142, p<0.0001 ;+\dagger F=4.35 \text {, } \\
\text { amination. }\end{array}$ & $\begin{array}{l}\text { athy and Diagnostic Clinical } \\
0.01 ; \neq \mathrm{F}=22.5, \mathrm{df}=3,146, \\
f=3,143, \mathrm{p}<0.01 .\end{array}$ & $\begin{array}{l}\text { teria for Apathy. Diagnoses of } \\
0.0001 ; \xi \mathrm{F}=22.1, \mathrm{df}=3,146, \mathrm{p}\end{array}$ & $\begin{array}{l}\text { lepression were made with the } \\
<0.0001 ; \uparrow \mathrm{F}=40.2, \mathrm{df}=3,146 \text {, }\end{array}$ \\
\hline
\end{tabular}

higher scores on the apathy factor were significantly correlated with higher apathy and anxiety scores), and MMSE scores $\left(\mathrm{R}^{2}=0.04, \mathrm{p}<0.01\right)$ (that is, higher scores on the apathy factor were significantly correlated with lower MMSE scores). Factor 2 (sadness/anxiety) showed a significant overall correlation $\left(\mathrm{R}^{2}=0.27, \mathrm{df}=3,127, \mathrm{p}<0.0001\right)$, but only anxiety scores explained a significant part of the variance $\left(R^{2}=0.25, p<0.0001\right)$.

\section{Overlap of apathy and depression in dementia}

The question also arises as to whether a diagnosis of apathy may "artificially" increase depression scores. To address this issue we calculated a two way ANOVA including apathy and major depression diagnoses as the grouping variables, and total scores on the Hamilton Depression Rating Scale as the dependent variable. There was the expected significant effect for depression $(\mathrm{F}=85.2, \mathrm{df}=1,108, \mathrm{p}<0.0001)$ and $\mathrm{a}$ significant effect for apathy $(\mathrm{F}=4.96, \mathrm{df}=1,108, \mathrm{p}<0.05)$, but no significant depression by apathy interaction $(\mathrm{F}=0.72$, $\mathrm{df}=1,108, \mathrm{p}=0.39$ ), demonstrating that the coexistence of apathy and depression does not produce higher depression scores than depression only. Finally, a stepwise regression analysis with Hamilton depression scores as the dependent variable and scores of apathy (caregiver version), anxiety, MMSE, age, and scores on the sad mood item of the SCID (that is, ratings of 1 (no depressed mood), 2 (questionable depressed mood), and 3 (definite depressed mood)) as the independent variables showed a significant overall effect $\left(R^{2}=0.68, \quad F=79.8, p<0.0001\right)$, and the variables that explained a significant part of the variance were anxiety $(53 \%)$, apathy $(13 \%)$, and sadness $(2 \%)$.

\section{DISCUSSION}

We demonstrated the reliability and validity of the Structured Interview for Apathy, and we examined the overlap between the constructs of apathy and depression in dementia. Apathy was present in $19 \%$ of patients, and $62 \%$ of the patients with apathy were also depressed. On the other hand, only $28 \%$ of the patients with depression had comorbid apathy. A factor analysis on the Hamilton Depression Rating Scale demonstrated a factor accounting for $33 \%$ of the variance that strongly correlated with scores on the Apathy Scale. Apathy was also diagnosed in a group of patients without syndromal depression, and a diagnosis of apathy had a minor impact on the rating of severity of depression in dementia. Finally, apathy, but not depression, was significantly associated with more severe cognitive deficits.

Before further comments, several limitations of our study should be pointed out. First, we do not have follow up data on our sample, and the temporal consistency of the diagnosis of apathy will have to be examined in future studies. Second, we have no pathological confirmation of our clinical diagnosis of probable AD. However, the NINCDS-ADRDA criteria have been demonstrated to have high sensitivity and specificity for the diagnosis of probable AD.

Whereas the negative impact of depression and apathy on the quality of life of patients with dementia and their respective caregivers has been consistently replicated, consensus as to how to diagnose these mood and behavioural changes has only recently emerged. ${ }^{15-20}$ Several studies demonstrated the validity of symptoms of depression in $\mathrm{AD}$, and specific diagnostic criteria have been proposed. ${ }^{21}$ The present study demonstrated that anxiety and apathy explain $65 \%$ of the variance with depression scores, whereas sadness only accounted for $2 \%$ of the variance. This finding suggests that, at least in dementia, depression may primarily result from a combination of symptoms of anxiety and apathy. On the other hand, the nosological position of apathy remains obscure, with some studies suggesting that apathy and depression are independent constructs, and other studies showing a significant overlap between apathy and depression. Frisoni and colleagues ${ }^{22}$ and Aalten and colleagues ${ }^{23}$ calculated a factor analysis on the Neuropsychiatric Inventory scores from $\mathrm{AD}$ patients and found that both apathy and depressed mood loaded on a single factor. On the other hand, using the same instrument and analytic technique, Fuh and coworkers $^{24}$ found that apathy and depression loaded on two independent factors. Strauss and Sperry ${ }^{16}$ examined a series of $100 \mathrm{AD}$ patients with an informant based assessment of apathy, and found no significant association between apathy and depression. de Jonghe and coworkers ${ }^{25}$ assessed a series of 281 patients using the Cambridge Examination for Mental Disorders of the Elderly (CAMDEX) and found that apathy and depression loaded on two independent factors. Our study 
addressed this issue using structured psychiatric interviews, standardised diagnostic criteria, and specific rating scales for both apathy and depression. A factor analysis of the Hamilton Depression Rating Scale produced two factors: the first included psychomotor retardation, loss of interest, poor energy, agitation, and poor appetite, and was construed as an apathy factor. The second factor included sad mood, guilt and suicidal ideation, anxiety, and insomnia, and was construed as a sadness/anxiety factor. We found significant positive correlations between the apathy factor and scores on the Apathy Scale, and between the sadness/anxiety factor and scores of anxiety and apathy. This finding is similar to the report by Marin $e a^{26}$ of a significant positive correlation between apathy scores and the Hamilton Depression Scale cluster of "apathy" symptoms (loss of interest, psychomotor retardation, lack of energy, and loss of insight).

The frequency of apathy in our patients with major depression was significantly higher than in patients with minor depression $\left(43 \% \vee 14 \%, \chi^{2}=6.81, \mathrm{df}=1, \mathrm{p}<0.01\right)$, suggesting different mechanisms for these disorders in $\mathrm{AD}$. Alternatively, major depression with apathy could be a more severe type of depression. In a previous study on a different sample of patients with $\mathrm{AD}$, we found that depressive symptoms started before the onset of cognitive deficits in about half of the patients with major depression, and the frequency of major depression was similar across the stages of $\mathrm{AD}^{27}$ On the other hand, depressive symptoms started after the onset of cognitive deficits in $90 \%$ of patients with dysthymia, the frequency of dysthymia was significantly higher in the early stages of dementia, and dysthymia was associated with a better awareness of functional impairments as compared to patients with either major or no depression. Based on these findings we suggested that dysthymia in $\mathrm{AD}$ could be a negative emotional reaction to the progressive cognitive decline, whereas major depression in $\mathrm{AD}$ could be more strongly related to biological factors. We also found a significant correlation between more severe apathy (but not depression) and more severe cognitive deficits. Similar associations between apathy and cognitive deficits have been reported in healthy elderly individuals and in patients with AD. ${ }^{15} 28$

The frequency of apathy in the present study (19\%) is lower than in other recent studies (ranging from $44 \%$ to $80 \%),{ }^{22-25}$ and several methodological differences may explain these discrepancies. Most other studies used broad behavioural symptom inventories, ${ }^{22-25}$ whereas we used a structured interview that was specifically designed to assess apathy in AD. Moreover, whereas most other studies diagnosed apathy based on cut off scores on ad hoc scales, ${ }^{29-31}$ we diagnosed apathy using standardised criteria that have been validated for the diagnosis of apathy in AD. Another important difference is that the mean MMSE score for our sample (23 points) was higher than in previous studies (with mean MMSE scores ranging from 13 to 18 points). ${ }^{22-25}$ Given that the frequency of apathy increases with the severity of dementia, the lower frequency of apathy in the present study may be related to the relatively less severe dementia in our sample as compared to previous studies.

In conclusion, we demonstrated the reliability and validity of the Structured Interview for Apathy in AD. We found that most patients with dementia and apathy had concomitant depression, but less than one third of depressed patients had concomitant apathy. Apathy in $\mathrm{AD}$ has been consistently associated with relatively more severe cognitive deficits, more severe impairments in activities of daily living, higher levels of burden and distress in caregivers, and increased resource utilisation. ${ }^{4}$ The use of specific structured interviews and valid diagnostic criteria to assess and diagnose apathy in $\mathrm{AD}$ will improve the identification of more homogeneous behavioural phenotypes and may help to clarify the mechanisms of these disorders.

\section{ACKNOWLEDGEMENTS}

We thank Janus Kremer, MD, Alicia Lischinski, MD, and Gustavo Petracca, MD for their contribution to the study, and Professor Assen Jablensky for his advice.

\section{Authors' affiliations}

S E Starkstein, Raul Carrea Institute of Neurological Research, Buenos Aires, Argentina

L Ingram, Fremantle Hospital, Fremantle, Australia

M L Garau, Departamento de Neurologia, Hospital Fernandez, Buenos Aires, Argentina

R Mizrahi, PET Center for Addiction and Mental Health, Clarke Division, Toronto, Canada

This study was partially supported with grants from the University of Western Australia, the Australian Rotary Health Research Fund, the Raine Medical Research Foundation, and the Fremantle Hospital Research Foundation.

Competing interests: none declared

Ethics approval: the protocol for this study was approved by the institutional Ethics Committee.

\section{REFERENCES}

1 Inwood B. Ethics and human action in early Stoicism. Oxford: Clarendon Press, 1985.

2 Berrios GE. The history of mental symptoms. Cambridge: Cambridge University Press, 1996.

3 Kraepelin E. Manic depressive insanity and paranoia. Salem, NH: Ayer, 1987.

4 Landes AM, Sperry SD, Strauss ME, et al. Apathy in Alzheimer's disease. J Am Geriatr Soc 2001;49(12):1700-7.

5 Olin JT, Schneider LS, Katz IR, et al. Provisional diagnostic criteria for depression of Alzheimer disease. Am J Geriatr Psychiatry 2002;10(2):125-8.

6 McKhann G, Drachman D, Folstein M, et al. Clinical diagnosis of Alzheimer's disease: report of the NINCDS-ADRDA Work Group under the auspices of Department of Health and Human Services Task Force on Alzheimer's Disease. Neurology 1984;34(7):939-44.

7 Spitzer RL, Williams JB, Gibbon M, et al. The Structured Clinical Interview for DSM-III-R (SCID). I: History, rationale, and description, Arch Gen Psychiatry 1992;49(8):624-9.

8 Folstein MF, Folstein SE, McHugh PR. "Mini-mental state". A practical method for grading the cognitive state of patients for the clinician. J Psychiatr Res 1975; 12(3): 189-98.

9 Hamilton M. A rating scale for depression. J Neurol Neurosurg Psychiatry 1960;23:56-62.

10 Hamilton MA. The assessment of anxiety state by rating. $\mathrm{Br} J$ Med Psychol 1959;32:50-5.

11 Hughes CP, Berg L, Danziger WL, et al. A new clinical scale for the staging of dementia. Br J Psychiatry 1982;140:566-72.

12 Starkstein SE, Migliorelli R, Manes F, et al. The prevalence and clinical correlates of apathy and irritability in Alzheimer's disease. Eur J Neurol 1995;2:540-6.

13 Starkstein SE, Petracca G, Chemerinski E, et al. Syndromic validity of apathy in Alzheimer's disease. Am J Psychiatry 2001;158(6):872-7.

14 Marin RS. Apathy: a neuropsychiatric syndrome. J Neuropsychiatry Clin Neurosci 1991;3(3):243-54

15 Boyle PA, Malloy PF, Salloway S, et al. Executive dysfunction and apathy predict functional impairment in Alzheimer disease. Am J Geriatr Psychiatry 2003;11(2):214-21.

16 Strauss ME, Sperry SD. An informant-based assessment of apathy in Alzheimer disease. Neuropsychiatry Neuropsychol Behav Neurol 2002;15(3): 176-83.

17 McPherson S, Fairbanks L, Tiken S, et al. Apathy and executive function in Alzheimer's disease. J Int Neuropsychol Soc 2002;8(3):373-81.

18 Robert PH, Clairet S, Benoit $M$, et al. The apathy inventory: assessment of apathy and awareness in Alzheimer's disease, Parkinson's disease and mild cognitive impairment. Int J Geriatr Psychiatry 2002;17(12):1099-105.

19 Ready RE, Ott BR, Grace J, et al. Apathy and executive dysfunction in mild cognitive impairment and Alzheimer disease. Am J Geriatr Psychiatry 2003; 11(2):222-8.

20 Marin RS, Butters MA, Mulsant BH, et al. Apathy and executive function in depressed elderly. J Geriatr Psychiatry Neurol 2003;16(2):1 12-6.

21 Olin JT, Katz IR, Meyers BS, et al. Provisional diagnostic criteria for depression of Alzheimer disease: rationale and background. Am J Geriatr Psychiatry 2002;10(2): 129-41.

22 Frisoni GB, Rozzini L, Gozzetti A, et al. Behavioral syndromes in Alzheimer's disease: description and correlates. Dement Geriatr Cogn Disord 1999; 10(2):130-8. 
23 Aalten $\mathbf{P}$, de Vugt ME, Lousberg R, et al. Behavioral problems in dementia: a factor analysis of the neuropsychiatric inventory. Dement Geriatr Cogn Dis 2003;15(2):99-105.

24 Fuh JL, Liu CK, Mega MS, et al. Behavioral disorders and caregivers' reaction in Taiwanese patients with Alzheimer's disease. Int Psychogeriatr 2001;13(1):121-8.

25 de Jonghe JF, Goedhart AW, Ooms ME, et al. Negative symptoms in Alzheimer's disease: a confirmatory factor analysis. Int J Geriatr Psychiatry 2003; 18(8):748-53.

26 Marin RS, Firinciogullari S, Biedrzycki RC. Group differences in the relationship between apathy and depression. J Nerv Ment Dis 1994; 182(4):235-9.
27 Migliorelli R, Teson A, Sabe L, et al. Prevalence and correlates of dysthymia and major depression among patients with Alzheimer's disease. Am J Psychiatry 1995; 152:37-44.

28 Lampe IK, Kahn RS, Heeren TJ. Apathy, anhedonia, and psychomotor retardation in elderly psychiatric patients and healthy elderly individuals. $J$ Geriatr Psychiatry Neurol 2001;14(1):11-6.

29 Burns A, Folstein S, Brandt J, et al. Clinical assessment of irritability, aggression, and apathy in Huntington and Alzheimer disease. J Nerv Ment Dis 1990;178(1):20-6.

30 Mega MS, Cummings JL, Fiorello T, et al. The spectrum of behavioral changes in Alzheimer's disease. Neurology 1996;46(1):130-5.

31 Levy ML, Cummings JL, Fairbanks LA, et al. Apathy is not depression. J Neuropsychiatry Clin Neurosci 1998;10(3):314-9.

\section{NEUROLOGICAL PICTURE}

\section{Ventriculitis and hydrocephalus: an unusual presentation of toxoplasmosis in an adult with human} immunodeficiency virus

\begin{abstract}
A 33 year old African man presented with a history of fever, confusion, and headache. An magnetic resonance imaging (MRI) scan revealed hydrocephalus, marked enhancement within the ventricular walls, and extensive confluent T2-weighted/fluid attenuated inversion recovery (FLAIR), high signal around the $3^{\text {rd }}$ and lateral ventricles, as well as within the splenium of the corpus callosum. Bilateral extra-ventricular drains were inserted. Cerebrospinal fluid (CSF) examination revealed: $28 / \mathrm{mm}^{3}$ lymphocytes, protein $2557 \mathrm{mg} / \mathrm{l}$, glucose $1.8 \mathrm{mmol} / \mathrm{l}$ (serum $6.3 \mathrm{mmol} / \mathrm{l}$ ), no organisms, and polymerase chain reaction (PCR) negative for tuberculosis (TB), cytomegalovirus (CMV), herpes simplex virus (HSV), and JC virus, but positive for Epstein-Barr virus (EBV). An human immunodeficiency virus (HIV)-1 enzyme-linked immunosorbent assay (ELISA) was positive with a CD4 count of $158 / \mathrm{mm}^{3}$. Serum CMV IgG and Toxoplasma IgG were positive consistent with previous exposure.

Treatment was commenced with anti-tuberculous medication, Aciclovir, and Ganciclovir. Despite this, he deteriorated and died a few days later. Post-mortem examination revealed extensive necrotising toxoplasmosis along the ventricular walls.

The radiological differential diagnosis of ventriculitis in HIV infection is usually that of TB, CMV, or lymphoma. ${ }^{1}$ In the context of HIV disease, lesions in the corpus callosum are most often seen in lymphoma and rarely in toxoplasmosis. ${ }^{2}$ Positive EBV PCR is also thought to be both highly sensitive and specific for primary central nervous system (CNS) lymphoma. ${ }^{3}$ Cerebral toxoplasmosis in the immunocompromised characteristically presents with mass lesions and although hydrocephalus is a common manifestation in congenital toxoplasmosis, it is rarely seen in adults. ${ }^{4}$ Our case stresses the importance that in HIV disease, neuroradiological and laboratory investigations can be grossly misleading, and that a trial of empirical anti-toxoplasmosis treatment should be considered for any CNS lesion in a deteriorating patient.
\end{abstract}

T de Silvo M Raychaudhuri M Poulton The Caldecot Centre, King's Healthcare NHS Trust, London, UK
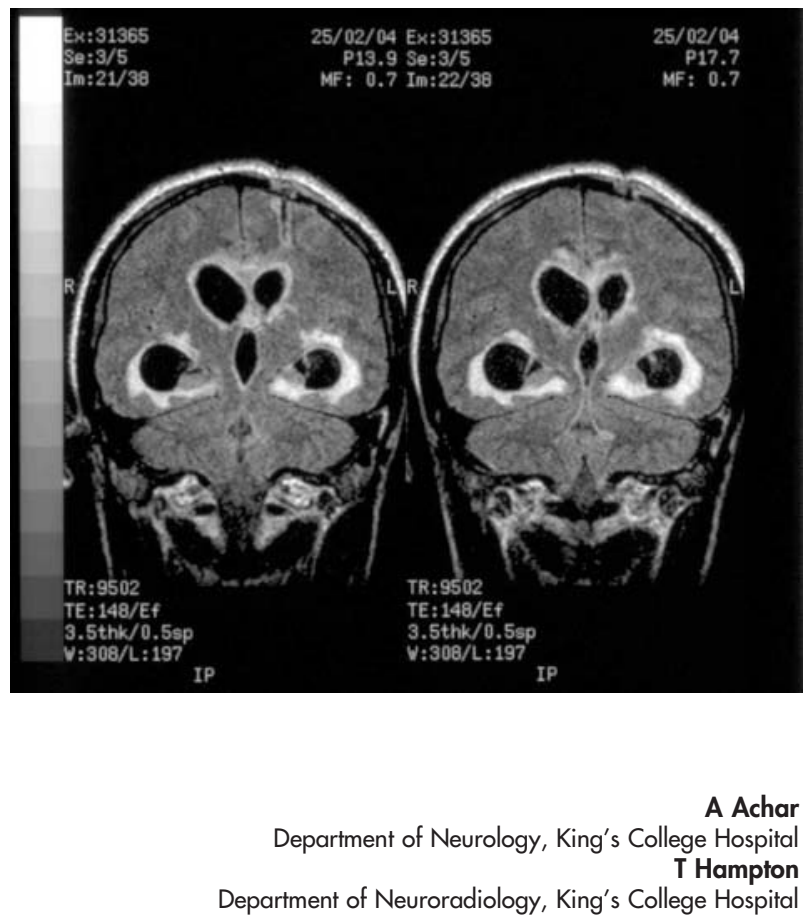

Correspondence to: $\operatorname{Dr} M$ Poulton, Consultant in HIV Medicine, The Caldecot Centre, King's Healthcare NHS Trust, 15-22 Caldecot Road, London SE5 9RS, UK; Mary.Poulton@kingsch.nhs.uk

\section{References}

1 Guerini $\mathrm{H}$, Helie $\mathrm{O}$, Leveque $\mathrm{C}$, et al. Diagnosis of periventricular ependymal enhancement in MRI in adults. J Neuroradiol 2003;30:46-50.

2 Lee HJ, Williams R, Kalnin A, et al. Toxoplasmosis of the Corpus Callosum: Another Butterfly. Am J Roetgenol 1996;166:1280-1.

3 Antinori A, Ammassari A, De Luca A, et al. Diagnosis of AIDS-related focal brain lesions: A decision-making analysis based on clinical and neuroradiologic characteristics combined with polymerase chain reaction assays in CSF. American Academy of Neurology 1997;48:687-94.

4 Lorberboym M, Wallach F, Estok L, et al. Rapid Differential Diagnosis of Cerebral Toxoplasmosis and Primary Central Nervous System Lymphoma by Thallium-201 SPECT. Journal of Nuclear Medicine 1996;37:1150-4. 